Final Beam Emittances at the end of $\bar{p}$-Accumulation for the Fermilab $\overline{\mathrm{P}}$ Source

K. Takayama and A. G. Ruggiero

Fermilab, April, 1981

CONTENTS

1. Introduction

2. $\overline{\mathrm{P}}$ Beam affected by Electron Cooling and Intra-Beam Scattering

2-a Equations of motion for an individual particle

2-b Variations of emittances due to cooling

2-c Distribution on the emittance space

2-d Evolution equations for emittances

2-e Difference equations

3. Results---Applications to the Fermilab Electron Cooling Ring $(200,400 \mathrm{MeV})-\cdots$

Appendix

A-1 Stability of the Euler Method

A-2 Linear tune shift due to space charge force of $\bar{p}$ beam References 
1. Introduction

The scheme to produce $\bar{p}$ at Fermilab includes an Accumulator where antiprotons, are RF stacked and cooled with the electron

cooling technique. For several conditions discussed in the design report', the beam energy in the accumulater $r$ ing is chosen to be several hundred millions of electron volts.

Toward the end of the process, when large number of $\bar{p}$ have been accumulated $\left(N_{\bar{p}}=10^{\prime \prime}\right)$, two major effects counteract each other in determining the final beam emittances: one is the $e^{-}$-cooling itself which would cause the emittances to match the e-beam temperature, the other is the intra-beam. scattering which would cause a decrease of the final density. Both these effects result from the Coulomb interaction among particles of the same charge. The e-beam cooling, though is localized in a small region where as the intra-beam scattering is spread all around the storage ring.

If we assume that the motion of a particle looks like that of a harmonic ocillator, we can regard a $\bar{p}$ beam as an isolated system of $\mathbf{N}$ three-dimensional harmonic oscillators ${ }^{(2)}$ which experience many random collisions among themselves and periodically take a "cold electron bath", as represented schematically in Fig. 1.

The phase space volume of such a system decreases due to collision with "cold" electrons in the cooling region. But in the rest of the storage ring it will increase due to the interaction among themselves. We expect an equilibrium for the volume of the system( $\bar{p}$ gas) will be reached over sometime characteristic of the 
Fig. 1
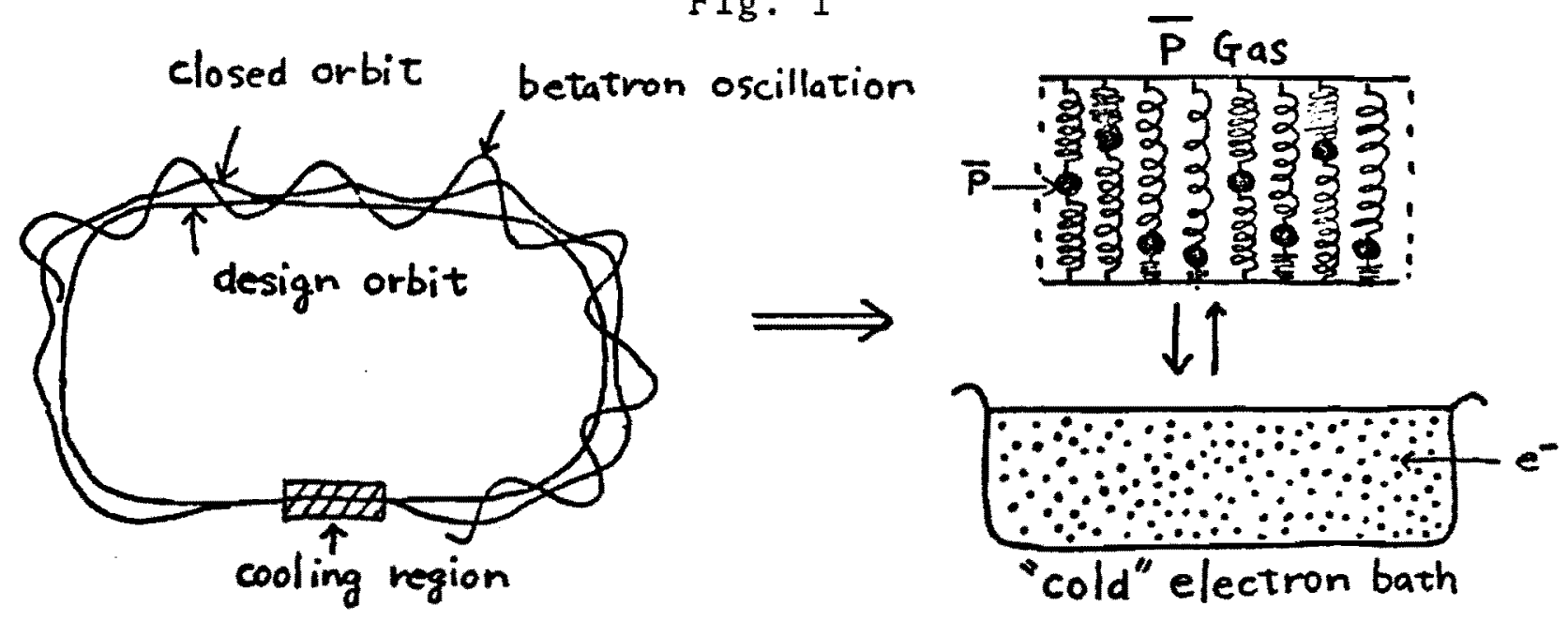

two processes we have described.

In following subsections $2-a \sim 2-d$, we discuss the behavior of

an individual particle in the emittance space and derive the evolution equations for beam emittances. In subsection 2-e, numerical methods to solve non-linear evolution equations are - exposed. In the final section, we will obtain the equilibrium emittances and the relaxation times, that are the times it takes for the $\bar{p}$ beam to reach the equilibrium, for different ring parameters. We shall cosider only the case of an unbunched beam.

2. $\overline{\mathrm{P}}$ Beam affected by Electron Cooling and Intra-Beam Scattering 2-a Equations of motion for an individual paticle

Taking into account both effects of collisions with "cold" electrons and other $\overline{\mathrm{p}}^{\prime} \mathrm{s}$, we can write equations of motion for an individual particle as follows: $\left.x_{i}^{\prime \prime}+k_{n}(s) x_{i}=\Omega(s) \delta_{i}+\sum_{i} D_{\bar{p}} \delta\left(x_{\bar{p}}\right)_{i}-\left(x_{e}\right)_{k}\right)_{z}+\sum_{j=1}^{N} I_{\bar{p} \bar{p}}\left(\left(x_{\bar{p}}\right)_{i}-\left(x_{\bar{p}}\right)_{j}\right)_{x}$ $\begin{aligned} y_{i}^{\prime \prime}+k_{y}(s) y_{i} & =\quad \sum_{k} D_{\overline{p e}}\left(\left(x_{\bar{p}}\right)_{i}-\left(x_{e}\right)_{k}\right)_{y}+\sum_{j=1}^{N} I_{\overline{p p}}\left(\left(x_{p}\right)_{i}-\left(x_{\bar{p}}\right)_{j}\right)_{y} \\ \dot{\delta}_{i} & =\quad \sum_{k} D_{\bar{p} e}\left(\left(k_{\bar{p}}\right)_{i}-\left(x_{e}\right)_{k}\right)_{s}+\sum_{j=1}^{N} I_{\overline{p p}}\left(\left(x_{p}\right)_{i}-\left(x_{p}\right)_{j}\right)_{s}\end{aligned}$ 
with $\quad x_{\bar{p}}=(x, y, \delta)$

where the index " $i$ " refers to the $i-t h \bar{p}$, a prime meansthe orbit derivative, a dot means the time derivative, $K_{x}(s)$ and $K_{Y}(s)$ are

the periodic lattice functions for horizontal and vertical directions, $\Omega(s)$ is the periodical bending function, $x$ and $y$ are the excursions from the design orbit for horizontal and vertical directions, $\delta$ is the momentum deviation $(\equiv \Delta \mathrm{p} / \mathrm{p})$, $D_{\bar{p} e}\left(\left(x_{\bar{p}}\right)_{i}-\left(x_{k}\right)_{k}\right)$ is the coulomb force due to the interaction with the $k$-th electron, and $I_{\bar{p} \bar{p}}\left(\left(x_{\bar{p}}\right)_{i}-\left(*_{\bar{p}}\right)_{j}\right)$ is the Coulomb force due to the interaction with the $j-$ th $\bar{p}$.

The analysis of Eq. (2-1) taking into account both effects has never been attempted before. It is possible to transform the e-beam interaction terms in Eq. (2-1) as equivalent to a friction force. The second terms, caused by intera-beam scatterings, have been approximated by Piwinski in a set of equations which relate

beam height, width and momentum spread.

Our goal is to combine the two approaches together, so we could ivestigate the behavior of a $\overline{\mathrm{p}}$ beam under both effects. To do so, we choose first to derive the variation of betatron emittances and longitudinal emittance (the momentum deviation) of a single particle after one pass through the cooling region.

2-b Variation of single particle emittances due to $\bar{e}$-cooling

In the linear approximation, the excursion of a test particle from the design orbit is described by two terms

$$
z \equiv z(s)=z_{\beta}(s)+\eta_{s}(s) \cdot \delta
$$


where $z$ stands for either $x$ or $y$ as we shall always adopt in the following. In particular the betatron motion around the closed orbit $\left(\eta_{g}(s) \cdot \delta\right)$ is well known to have an invariant

$$
J_{z}=\frac{1}{2}\left(\gamma_{z}(s) z_{\beta}^{2}+2 \alpha_{z}(s) z_{\beta} P_{z_{\beta}}+\beta_{z}(s) P_{z_{\beta}}^{2}\right)
$$

One obtains also an invariant for the longitudinal motion. In the case of an unbunched beam, one chooses

$$
J_{\delta}=\delta^{2}
$$

In a typical electron cooling ring, the phase advance across the cooling region is usually small, and the orbit functions of $\beta_{z}, \eta_{z}$ and $\alpha_{z}$ do not change appreciably. Therefore these can be assumed as constant and will be denoted with an asterisk(*) to show their values in that region. Also, the effect of the

- Eriction force which the antiproton experiences as traversing the electron beam can be thought as lumped. The friction force components in the laboratory frame are:

$$
\begin{aligned}
& F_{z}=-\frac{4 \pi e^{4} n L}{m c^{2} \beta^{2} \gamma^{4}} \cdot \frac{z^{\prime}}{\left(\theta_{\perp}^{2}+x^{\prime^{2}}+y^{\prime 2}+\delta^{2} / \gamma^{2}\right)\left(\theta_{\perp}^{2}+\theta_{11}^{2} / \gamma^{2}+x^{\prime 2}+y^{\prime 2}+\delta^{2} / \gamma^{2}\right)^{1 / 2}} \\
& F_{s}=-\frac{4 \pi e^{4} n L}{m c^{2} \beta^{2} \gamma^{4}} \cdot \frac{\delta}{\left(\theta_{\perp}^{2}+\theta_{11}^{2} / \gamma^{2}+x^{\prime 2}+y^{\prime 2}+\delta^{2} / \gamma^{2} X \theta_{1 / \gamma^{2}} / \gamma^{2}+x^{\prime 2}+y^{\prime 2}+\delta^{2} / \gamma^{2}\right)^{1 / 2}}
\end{aligned}
$$

where $c$ is the velocity of light, $e$ is the unit charge, $m$ is the rest mass of an electron, $\beta$ and $\gamma$ are the relativistic quantities of the electron, $n$ is the density of the electron beam, $I$ is the Coulomb $\log , \theta_{\perp}$ and $\theta_{4}$ are the relative spreads of the transverse and longitudinal velocities of the electron beam, $z^{*}\left(x^{\circ}\right.$ or $y^{\circ}$ ) and 
$\delta$ are the transverse and longitudinal momenta of the antiproton. The variations of the momentum components after one crossing are

$$
\begin{aligned}
\Delta P_{z} & =F_{z} l / M \gamma \beta^{2} c^{2} \\
\Delta \delta & =F_{s} l / M \gamma \beta^{2} c^{2}
\end{aligned}
$$

where $l$ is the length of the cooling region and $M$ is the rest mass of an antiproton.

Using the Eqs. (2-3), (2-4) for the emittances and Eqs. (2-7), $(2-8)$, we can easily derive the variations of the betatron and longitudinal emittances every turn. Here we assume $\eta^{*}=\eta^{*}=\alpha^{*}=0$ and neglect the second order terms for $\Delta \mathrm{p}_{\mathrm{z}}$ and $\Delta \delta^{(5)}$. We have

$$
\begin{aligned}
\Delta J_{z} & \simeq \beta_{z}^{*} P_{z p} \Delta P_{z} \\
& =\frac{\beta_{z}^{*} \ell P_{z p}}{M r \beta^{2} c^{2}} \cdot F_{z} \\
\Delta J_{s} & =(\delta+\Delta \delta)^{2}-\delta^{2} \\
& \simeq \frac{2 \ell \delta}{M \gamma \beta^{2} c^{2}} \cdot F_{s}
\end{aligned}
$$

Moreover putting Eqs.(2-5),(2-6) into Eqs.(2-9), (2-10) and averaging them over one betatron period, we obtain approximately the representations for variations of the betatron and longitudinal emittances

$$
\begin{aligned}
& \Delta J_{z}=-k J_{z y} / 2\left(\theta_{\perp}^{2}+\frac{J_{x}}{\beta_{x}^{*}}+\frac{J_{y}}{\beta_{y}^{*}}+\frac{J_{s}}{\gamma^{2}}\right)\left(\theta_{\perp}^{2}+\frac{\theta_{11}^{2}}{\gamma^{2}}+\frac{J_{x}}{\beta_{x}^{*}}+\frac{J_{y}}{\beta_{y}^{*}}+\frac{J_{s}}{\gamma^{2}}\right)^{\frac{1}{2}} \\
& \Delta J_{s}=-k J_{s} /\left(\theta_{\perp}^{2}+\frac{\theta_{11}^{2}}{\gamma^{2}}+\frac{J_{x}}{\beta_{x}^{*}}+\frac{J_{y}}{\beta_{y}^{*}}+\frac{J_{s}}{\gamma^{2}}\right)\left(\frac{\theta_{11}^{2}}{\gamma^{2}}+\frac{J_{x}}{\beta_{x}^{*}}+\frac{J_{y}}{\beta_{y}^{*}}+\frac{J_{s}}{\gamma^{2}}\right)^{\frac{1}{2}}
\end{aligned}
$$

with

$$
K=\frac{8 \pi r_{e} r_{p} L J_{e} l}{c e(\beta \gamma)^{5}}
$$


, where $r_{e}$ and $r_{p}$ are the classical radius of an electron and an antiproton, $J_{e}$ is the current density of the electron beam.

Next, averaging $\Delta J_{Z}$ and $\Delta J_{s}$ over all particles, we obtain the variations of beam emittances. For this purpose we need the distribution function of particles on the emittance space.

2-c Distribution on the emittance space

The distribution function on the emittance space will be obtained according to the following flow chart:

\section{Flow Chart}

$\rho$ : distribution function in the real space at the cross section

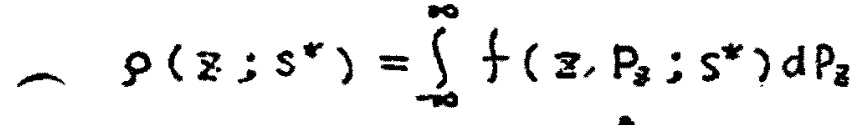

$$
\begin{aligned}
& z=\sqrt{2 J_{z} \beta_{z}^{*}} \sin \psi_{z} \\
& P_{z}=\sqrt{2 J_{z} / \beta_{z}^{*}} \cos \psi_{z}
\end{aligned}
$$

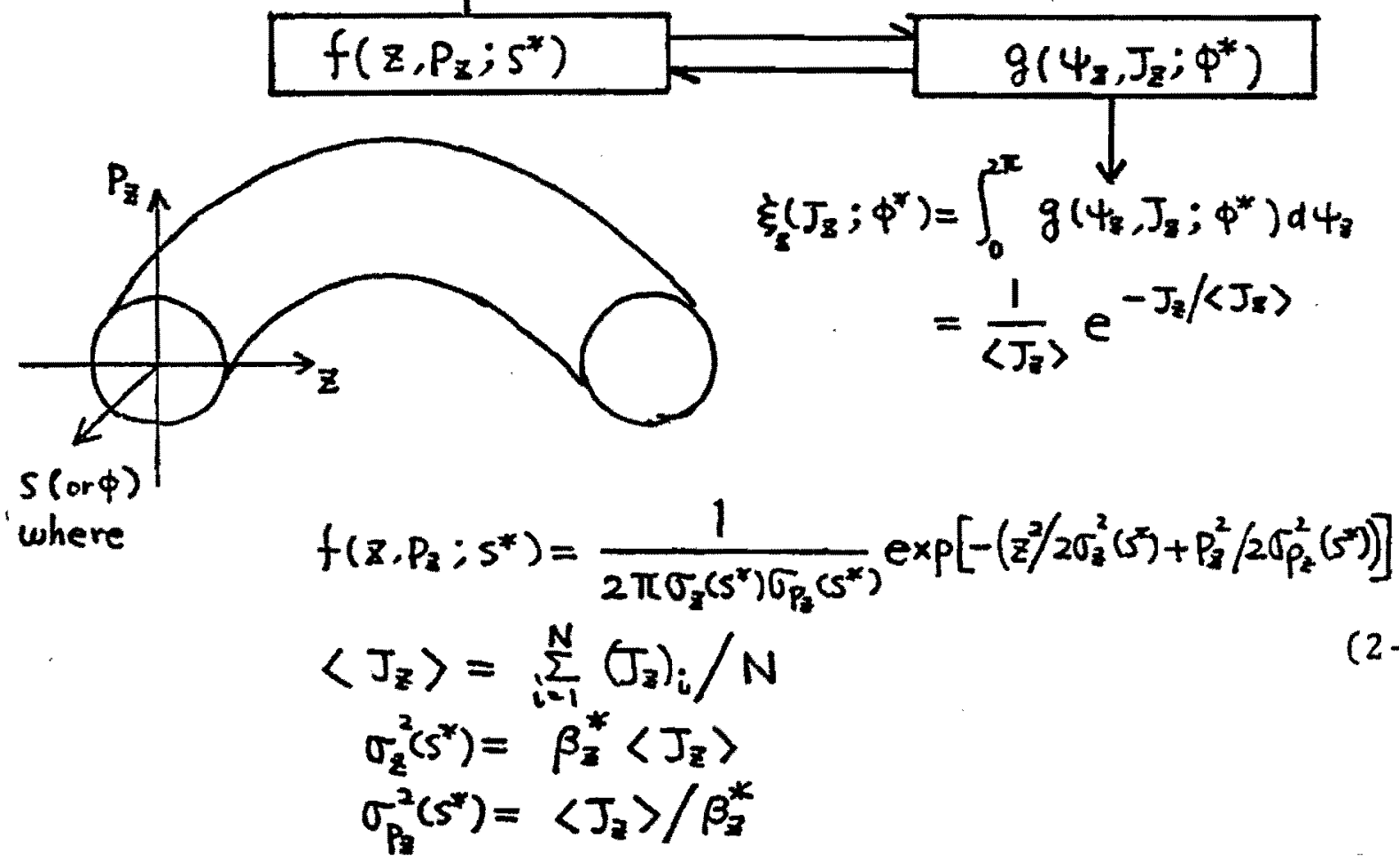


Namely the transformation into action-angle variables $\left(J_{z}, \Psi_{z}\right)$ leads to the distribution function

$$
g\left(J_{z}, \psi_{2} ; \phi^{*}\right)=\frac{1}{2 \pi\left\langle J_{z}\right\rangle} e^{-J_{z} /\left\langle J_{z}\right\rangle}
$$

From Eq. (2-15), we can write the beam distribution function on the betatron emittance space $\xi_{z}$ at the cross section $\phi^{*}$ of the torus

$$
\xi_{z}\left(J_{z} ; \phi^{*}\right)=\frac{1}{\left\langle J_{z}\right\rangle} e^{-J_{z} /\left\langle J_{z}\right\rangle}
$$

In the similar way, the distribution function on the longitudinal emittance space is obtained. We write the distribution function $\xi_{s}\left(J_{s} ; t\right)$ as follows:

$$
\xi_{s}\left(J_{s} ; t\right)=\frac{1}{\sqrt{2 \pi\left\langle J_{s}\right\rangle}} \cdot \frac{1}{\sqrt{J_{s}}} e^{-J_{s} / 2\left\langle J_{s}\right\rangle}
$$

2-e Evolution equations for emittances

Let us aerage the variation of emittances per revolution Eqs.(2-11),(2-12) over the distributions on the betatron and longitudinal emittance space. We write them as follows:

$$
\begin{aligned}
& \Delta\left\langle J_{s}\right\rangle=\frac{-k}{\left.\left\langle J_{x} \times J_{y}\right\rangle \sqrt{2 \pi\left\langle J_{s}\right.}\right\rangle} \iiint_{0}^{\infty} \frac{\sqrt{J_{s}} \exp \left[-\left(J_{x} /\left\langle J_{x}\right\rangle+J_{y} \mid\left\langle J_{y}\right\rangle+J_{s} / 2\left\langle J_{s}\right\rangle\right)\right] d J_{x} d J_{y} d J_{s}}{\left(\theta_{\perp}^{2}+\frac{\theta_{11}^{2}}{\gamma^{2}}+\frac{J_{x}}{\beta_{z}^{*}}+\frac{J_{y}}{\beta_{y}^{*}}+\frac{J_{s}}{\gamma^{2}}\right)\left(\frac{\theta_{u t}^{2}}{\gamma^{2}}+\frac{J_{x}}{\beta_{x}^{*}}+\frac{J_{y}}{\beta_{y}^{*}}+\frac{J_{s}}{\gamma^{2}}\right)^{1 / 2}} \\
& \Delta\left\langle J_{z}\right\rangle=\frac{-K}{\left.2\left\langle J_{x} X J_{y}\right\rangle \sqrt{2 \pi\left\langle J_{s}\right.}\right\rangle} \iint_{0}^{\infty} \frac{J_{x} \exp \left[-\left(J_{x} /\left\langle J_{x}\right\rangle+J_{y} /\left\langle J_{y}\right\rangle+J_{s} / 2\left\langle J_{s}\right\rangle\right)\right] d J_{x} d J_{y} d J_{s}}{\sqrt{J_{s}}\left(\theta_{\perp}^{2}+\frac{J_{x}}{\beta_{x}^{*}}+\frac{J_{y}}{\beta_{y}^{*}}+\frac{J_{s}}{\gamma^{2}}\right)\left(\theta_{\perp}^{2}+\frac{\theta_{u}^{2}}{\gamma^{2}}+\frac{J_{x}}{\beta_{x}^{*}}+\frac{J_{y}}{\beta \beta_{y}^{*}}+\frac{J_{s}}{\gamma^{2}}\right)^{1 / 2}}
\end{aligned}
$$

Devide both sides of these equations by the revolution time $T_{0}$ to obtain the differential equations

$$
\begin{aligned}
& \frac{d\left\langle J_{s}\right\rangle}{d \tau} \simeq \frac{\Delta\left\langle J_{s}\right\rangle}{T_{0}}=C_{s}\left(\left\langle J_{x}\right\rangle,\left\langle J_{y}\right\rangle,\left\langle J_{s}\right\rangle\right) \\
& \frac{d\left\langle J_{z}\right\rangle}{d t} \simeq \frac{\Delta\left\langle J_{z}\right\rangle}{T_{0}}=C_{z}\left(\left\langle J_{x}\right\rangle,\left\langle J_{y}\right\rangle,\left\langle J_{s}\right\rangle\right) .
\end{aligned}
$$


This means that the electron cooling has been approximated as a damping force spread uniformly around the circumference of the ring.

Then if we use the representations for the rate of change of the betatron and longitudinal emittances due to intra-beam scattering, as derived by Piwinski, we know the behaviour of the anti-proton beam through the equations

$$
\begin{aligned}
& \frac{d\left\langle J_{s}\right\rangle}{d t}=C_{s}\left(\left\langle J_{x}\right\rangle,\left\langle J_{y}\right\rangle,\left\langle J_{s}\right\rangle\right)+2 A\left(1-\frac{\eta^{2}\left\langle J_{s}\right\rangle}{\sqrt{\beta_{x}\left\langle J_{x}\right\rangle+\eta^{2}\left\langle J_{s}\right\rangle}}\right) f_{1}(a, b, c) \cdot 2\left\langle J_{s}\right\rangle \\
& \frac{d\left\langle J_{x}\right\rangle}{d t}=C_{x}\left(\left\langle J_{x}\right\rangle,\left\langle J_{y}\right\rangle,\left\langle J_{s}\right\rangle\right)+A\left[f_{2}\left(\frac{1}{a}, \frac{b}{a}, \frac{c}{a}\right)+\frac{\eta^{2}\left\langle J_{s}\right\rangle}{\sqrt{\beta_{x}\left\langle J_{x}\right\rangle+\eta^{2}\left\langle J_{s}\right\rangle}} f_{1}(a, b, c)\right] \cdot 2\left\langle J_{x}\right\rangle \\
& \frac{d\left\langle J_{y}\right\rangle}{d t}=C_{y}\left(\left\langle J_{x}\right\rangle,\left\langle J_{y}\right\rangle,\left\langle J_{s}\right\rangle\right)+A f_{3}\left(\frac{1}{b}, \frac{a}{b}, \frac{c}{b}\right) \cdot 2\left\langle J_{y}\right\rangle
\end{aligned}
$$

with

$$
\begin{aligned}
& A=c r_{p}^{2} N / C_{0} 16 \pi \sqrt{\pi}\left\langle J_{x}\right\rangle\left\langle J_{y}\right\rangle \sqrt{\left\langle J_{s}\right\rangle} \beta^{3} \gamma^{3} \\
& a=\beta_{x}^{3 / 2} \sqrt{J_{s}} / \gamma \sqrt{\beta_{x}\left\langle J_{x}\right\rangle+\eta^{2}\left\langle J_{s}\right\rangle} \\
& b=\sqrt{\frac{\beta_{y}}{\left\langle J_{y}\right\rangle} \cdot \frac{\sqrt{\left\langle J_{s}\right\rangle}}{r} \cdot \frac{\beta_{x}\left\langle J_{x}\right\rangle}{\sqrt{\beta_{x}\left\langle J_{x}\right\rangle+\eta^{2}\left\langle J_{s}\right\rangle}}} \\
& c=\beta \gamma \sqrt{2 \frac{\bar{b}}{r_{p}}}, \quad \bar{b}=\frac{1}{2}(r / \rho)^{1 / 3} \quad(\rho \text { : volume density) }
\end{aligned}
$$

scattering function

$f(a, b . c)=2 \int_{0}^{\infty} \int_{0}^{\pi 2 \pi} \int_{0}^{2 \pi} \exp \left[-\rho\left(\cos ^{2} \mu+\left(a^{2} \cos ^{2} \nu+b^{2} \sin ^{2} \nu\right)-\sin ^{2} \mu\right) \ln \left(c^{2} \rho\right)\left(1-3 \cos ^{2} \mu\right) \sin \mu d \nu d \mu d \rho\right.$.

To solve Eqs. (2-21), we have to rely on the aid of a computer.

2-e Difference equations

We can solve a set of time dependent equations by the Euler 
method. If $\left\langle J_{x}\right\rangle,\left\langle J_{y}\right\rangle$ and $\left\langle J_{s}\right\rangle$ are given at step $n$, namely $\left\langle J_{x}\right\rangle_{n},\left\langle J_{y}\right\rangle_{n}$ and $\left\langle J_{s}\right\rangle_{n} \mid\left\langle J_{x}\right\rangle_{0},\left\langle J_{y}\right\rangle_{0}$ and $\left\langle J_{s}\right\rangle_{0}$ are defined as initial conditions), we wish to determine $\left\langle J_{x}\right\rangle,\left\langle J_{y}\right\rangle$ and $\left\langle J_{s}\right\rangle$ at step $n+1$. The simplest method is the Euler explicit first order

method,

$$
\begin{aligned}
& \left\langle J_{x}\right\rangle_{n+1}=\left\langle J_{x}\right\rangle_{n}+F_{x}\left(\left\langle J_{z}\right\rangle_{n},\left\langle J_{y}\right\rangle_{n},\left\langle J_{s}\right\rangle_{n}\right) \cdot \Delta t \\
& \left\langle J_{y}\right\rangle_{n+1}=\left\langle J_{y}\right\rangle_{n}+F_{y}\left(\left\langle J_{x}\right\rangle_{n},\left\langle J_{y}\right\rangle_{n},\left\langle J_{s}\right\rangle_{n}\right) \cdot \Delta \tau \\
& \left\langle J_{s}\right\rangle_{n+1}=\left\langle J_{s}\right\rangle_{n}+F_{s}\left(\left\langle J_{x}\right\rangle_{n},\left\langle J_{y}\right\rangle_{n},\left\langle J_{s}\right\rangle_{n}\right) \cdot \Delta t
\end{aligned}
$$

with

$$
\begin{aligned}
& F_{s}=C_{s}\left(\left\langle J_{x}\right\rangle_{n},\left\langle J_{y}\right\rangle_{n},\left\langle J_{s}\right\rangle_{n}\right)+2 A_{n}\left(1-\frac{\eta^{2}\left\langle J_{s}\right\rangle_{n}}{\sqrt{\beta_{x}\left\langle J_{x}\right\rangle_{n}+\eta^{2}\left\langle J_{s}\right\rangle_{n}}}\right) f_{1}\left(a_{n}, b_{n}, C_{n}\right) \cdot 2\left\langle J_{s}\right\rangle_{n} \\
& F_{x}=C_{x}\left(\left\langle J_{x}\right\rangle_{n},\left\langle J_{y}\right\rangle_{n},\left\langle J_{s}\right\rangle_{n}\right)+A_{n}\left[f_{z}\left(\frac{1}{a_{n}}, \frac{b_{n}}{a_{n}}, \frac{C_{n}}{a_{n}}\right)+\frac{\eta^{2}\left\langle J_{s}\right\rangle_{n}}{\sqrt{\beta_{x}\left\langle J_{x}\right\rangle_{n}+\eta^{2}\left\langle J_{s}\right\rangle_{n}}} f_{1}\left(a_{n}, b_{n}, C_{n}\right)\right] \cdot 2\left\langle J_{x}\right\rangle_{n} \\
& F_{s}=C_{y}\left(\left\langle J_{x}\right\rangle_{n},\left\langle J_{y}\right\rangle_{n},\left\langle J_{s}\right\rangle_{n}\right)+A_{n} f_{3}\left(\frac{1}{b_{n}} \cdot \frac{a_{n}}{b_{n}}, \frac{C_{n}}{b_{n}}\right) \cdot 2\left\langle J_{y}\right\rangle_{n}
\end{aligned}
$$

where $\Delta t$ is the time-step.

In the Euler method, the functions $F_{x}, F_{y}, F_{S}$ are evaluated only at time $t_{n}$, and hence the method is explicit and first-order accurate in the time-step $\Delta t$ only. Investigation of the stability of the method is discussed in Appendix. Each of the functions $F_{x}, F_{y}$ and $F_{S}$ includes two triple integrations, the first term corresponds to the effect of electron cooling and the second to that of intra-beam scattering.

The first triple integration is calculated by a numerical method. We like to remark the following facts: the integrand has 
a steep variation in the range of $0\langle\mathrm{~J} \leqslant 3\langle\mathrm{~J}\rangle$ and we found that as cooling proceeds, 〈J generally decreases by even more than an order of magnitude. Consequently, the mesh into which the emittance space $\left(J_{x}, J_{y}, J_{s}\right)$ is subdivided must be dense in the neighbour of the proximity and adjustable inside as cooling proceeds. The mesh therefore is chosen as follows:

$$
\Delta J=\left\{\begin{array}{rc}
\langle J\rangle / 20 & 0\langle J \leqq\langle J\rangle \\
\langle J\rangle / 10 & \langle J\rangle\langle J \leqq 2\langle J\rangle \\
\langle J\rangle / 10 & 2\langle J\rangle\langle J \leqq 4\langle J\rangle \\
& 4\langle J\rangle\left\langle J \leqq J_{\max }(=50\langle J\rangle)\right.
\end{array}\right.
$$

The value of the integrand at the center of each cubic mesh is calculated, and multiplied by the volume of the corresponding - cubic mesh. All the values so obtained are finally summed up.

The CERN Program Library "TRIINT" has been used to calculate the triple integrals corresponding to the intra-beam scattering term in Eq. (2-21). Actually a CERN version to estimate intra-beam scattering effects has been included in our own computer code "EVOLU".

3. Results---Application to the Fermilab electron cooling ring $(200,400 \mathrm{MeV})---$

One may suppose that, as $\bar{e}-$ cooling proceeds, the space charge force as the over-all effect of Coulomb interaction with other $\bar{p}^{\prime} s$ becomes to affect on the behavior of an individual particle. However, one must remind the following fact; although the beam 
size shrinks by cooling, the linear tune shift, which is regarded as the measure of the space charge force, does not change(see Appendix). Therefore, neglecting non-linear terms of the space charge force, one can consider the intra-beam scattering to be a dominant cause of beam blow-up.

Through the above mentioned calculation, we have assumed that the beam always remains the Gauss distribution. Strictly speaking, the non-linearity in the large amplitude regions of the friction force may brake the Gauss distribution of the $\bar{p}$ beam at the early stage of cooling. In the present study, we belive that the difference from the exact Gauss distribution is a little even at the early stage, since the initial velocity spreads of the $\bar{p}$ beam are chosen to be smaller values than that of the $e^{-}$beam.

The parameters used in the present numerical calculations are listed in Table 1 . Most of these parameters have been chosen in the design study of the Fermilab Electron Cooling Ring. But the relative spreads of the transverse and longitudinal velocities of the $\mathrm{e}^{-}$beam, that is, $\theta_{\perp}$ and $\theta_{11}$ have not been known exactly yet. Therefore we used the values obtained empirically from cooling experiments which have been proceeded elsewhere so far.

In Fig.2-a $\sim 2-c$, the solutions of difference evolution equations are shown as the function of time. As expected, beam emittances reach to an equilibrium state. And the behavior of the emittance curves in the region, where the longitudinal emittance has the minimum value, can be interpreted easily by the fact that rapid damping of the momentum spread enhances the intra-beam 
scattering at a streach.

The solutions of evolution equations without the intra-beam scattering terms are shown in the same figure(Fig.2-a). We can not find a large difference between two cases at the early stage of $e^{-}$cooling corresponding to the so-called e-fold time. We explain these results by the fact that the intra-beam scattering is dependent only upon the beam emittances.

Furthermore, we write the time, when the system takes to reach the equilibrium state, and its equilibrium emittances, in the terms of $\tau_{\text {relaxand }}\left(\left\langle J_{x}\right\rangle_{\text {find }},\left\langle J_{y}\right\rangle_{\text {find }},\left\langle J_{s}\right\rangle_{\text {fimal }}\right)$. So these values are plotted as a function of the electron beam current, in Figs. 2-d, 2-e.

Last let us refer to the dependence of both effects, i.e. the intra-beam scattering and $\mathrm{e}^{-c o o l i n g, ~ o n ~ t h e ~ b e a m ~ e n e r g y ~ b y ~}$ showing the numerical results for the beam energy of $400 \mathrm{MeV}$, in Fig.2-e. The remarkable difference of $\tau_{\text {relax }}$ between both cases of $200 \mathrm{MeV}$ and $400 \mathrm{MeV}$ results from the dependence of the friction force $F_{c}$ upon the beam energy: $F_{c} \propto(\beta \gamma)^{-5}$.

Consequently, we conclude that the electron cooling technique has the limit. Considering an allowable accumulating time and required beam emittances under the obtained results, one may choose the current density of the electron beam in a cooling $r$ ing. 
Table 1

Parameters of the lattice

Betatron amplitudes

$$
\begin{aligned}
\beta_{x}^{*}, \beta_{y}^{*} & =10 \mathrm{~m} \\
\ell & =10 \mathrm{~m}
\end{aligned}
$$

Cooling region length

Parameters of the electron beam

Electron current density

$\mathrm{J}_{e}=\cdot 2--1 \cdot \mathrm{A} / \mathrm{cm}^{2}$

Relative spread of $e^{-}$beam velocity

$$
\theta_{\perp}=2 . * 10^{-3}
$$$$
\theta_{11}=1 . * 10^{-4}
$$

Coulomb log.

$\mathrm{L}=15$

Parameters of the anti-proton beam

Number of accumulated $\bar{p}$

$$
\begin{aligned}
N & =1.5 * 10^{\prime \prime} \\
\left\langle J_{x}\right\rangle,\left\langle J_{y}\right\rangle & =7 . * 10^{-6}
\end{aligned}
$$

Beam emittances for transverse

(this value is corresponding to $\sqrt{6} \sigma$ beam emittance of

$42 \pi \mathrm{m}-\mathrm{mr} \mathrm{ad})$

Momentum deviation

$$
\begin{aligned}
\left\langle J_{s}\right\rangle \equiv \delta^{2} & =1 . * 10^{-6} \\
E & =200-400 \mathrm{MeV}
\end{aligned}
$$

Beam energy 
Appendix

A-1 Stability of the Euler Method

To investigate the stability of the method, it is assumed that a small error, $\epsilon_{n}$, exists at step $n$ and we question how the error is amplified to step $n+1$. To do so, the difference equation

$$
J_{n+1}=J_{n}+F\left(J_{n}\right) \cdot \Delta \tau
$$

is linearized about the small error,

$$
\begin{aligned}
& \left(J_{0}\right)_{n}+\epsilon_{n}+\left[F\left(\left(J_{0}\right)_{n}\right)+\left.\frac{\partial F}{\partial J}\right|_{\left.J=\sigma_{0}\right)_{n}} \cdot \epsilon_{n}\right] \cdot \Delta t \\
= & \left(J_{0}\right)_{n}+F\left(\left(J_{0}\right)_{n}\right) \cdot \Delta t+\epsilon_{n}+\left.\frac{\partial F}{\partial J}\right|_{J=\left(J_{0}\right)_{n}} \cdot \epsilon_{n} \cdot \Delta t
\end{aligned}
$$

, so that $\epsilon_{n+1}=\epsilon_{n}+\left.\frac{\partial F}{\partial J}\right|_{n} \cdot \epsilon_{n} \cdot \Delta t$

Here, we can define the amplification factor of the error

$$
g=1+\left.\frac{\partial F}{\partial J}\right|_{n} \cdot \Delta t
$$

The stability condition is $|g| \leqq 1$. clearly, for our evolution eqations

$$
\frac{\partial F}{\partial J}=-\frac{2}{\tau_{c}}+\frac{2}{\tau_{i}} \leqq 0
$$

where $\tau_{c}$ is the instantaneous damping time due to electron cooling and $\tau_{i}$ is the instantaneous rising time due to intra-beam scattering. The stability condition is satisfied if the time-step $\Delta t$ is sufficiently small such that

$$
\Delta t \leqq \frac{\tau_{c} \tau_{i}}{\tau_{i}-\tau_{c}}
$$

The values of the right-hand side of $\mathrm{Eq} \cdot(\mathrm{A}-4)$ at the cooling stage - are summarized in Table 2. 


\begin{tabular}{c|cc}
$t$ & early times & euilibrium state \\
\hline$-\frac{\tau_{c} \tau_{i}}{\tau_{i}-\tau_{c}} \simeq$ & several seconds & $\infty$ \\
\hline
\end{tabular}
reasonable value.

A-2 Linear tune shift due to space charge force of $\bar{p}$ beam

We consider the $\bar{p}$ beam with the beam size of the diameter a and $b$ for horizontal and vertical, corresponding to $\sqrt{2} \sigma$ beam.

The first order terms of the space charge potential are described as follows;

$$
V(x, y)=\frac{\pi p_{0} a b r_{p}}{\beta^{2} r^{3} e}\left(\frac{2}{a(a+b)} x^{2}+\frac{2}{b(a+b)} y^{2}\right)
$$

where $r_{p}$ is the classical radius of an antiproton, $\rho_{0}$ is the line density, $\beta$ and $\gamma$ are the relativistic quantities, and $e$ is the unit charge.

The effect of the potential Eq. (A-5) on the betatron oscillations is equivalent to a quadrupole magnet. Therefore it causes the so-called linear tune shifts. We can derive easily this linear tune shifts,

$$
\begin{aligned}
& \Delta \nu_{x}=\frac{\rho_{0} a b r_{p}}{\beta^{2} \gamma^{2} e} \frac{1}{a(a+b)} \int_{0}^{c_{0}} \beta_{x}(s) d s \\
& \Delta \nu_{y}=\frac{p_{0} a b r_{p}}{\beta^{2} \gamma^{2} e} \frac{1}{b(a+b)} \int_{0}^{c_{0}} \beta_{y}(s) d s
\end{aligned}
$$

As we can assume $a=b$, we know that, although the beam size shrinks, the linear tune shifts Eqs. $(A-6),(A-7)$ do not change. 


\section{References}

(1) "The Fermilab Antiproton Source Design Report ", Fermilab, April (1981)

(2) R. Takayama, " On an Invariant of a system affected by Intra-Beam Scattering ", FN Note 336 , (1981)

(3) T. Ogino and A. G. Ruggiero "The Physics of Electron Cooling ", Fermilab Internal Report, (1979)

(4) A. Piwinski, " Intra-Beam Scattering ", Proc. 9th Int. Conf. on High Energy Accelerator, p. 405 (1974)

(5) These second order terms would cause the $\bar{p}$ beam to reach an equilibrium with the e beam temperature. We expect this to be cosiderably smaller than the intra-beam scattering effects.

(6) Private communication from D. Möhl (1980)

We understand the CERN program "INTBMS" was written by F. Sacherer.

(7) B. W. Montague, CERN 68-38, p. 64(1968)

(8) D. E. Potter, "DIFFERENCE SCHEMES AND NUMERICAL ALGorithms ", Proc. Computing as a Language of Physics, IAEA, VIENNA, p.57 (1972)

(9) K. Takayama, "Difference Resonance Theory and Application to KEK P.S.", KEK Internal Note (1978)

(10) K. Takayama prepared the computer code. 


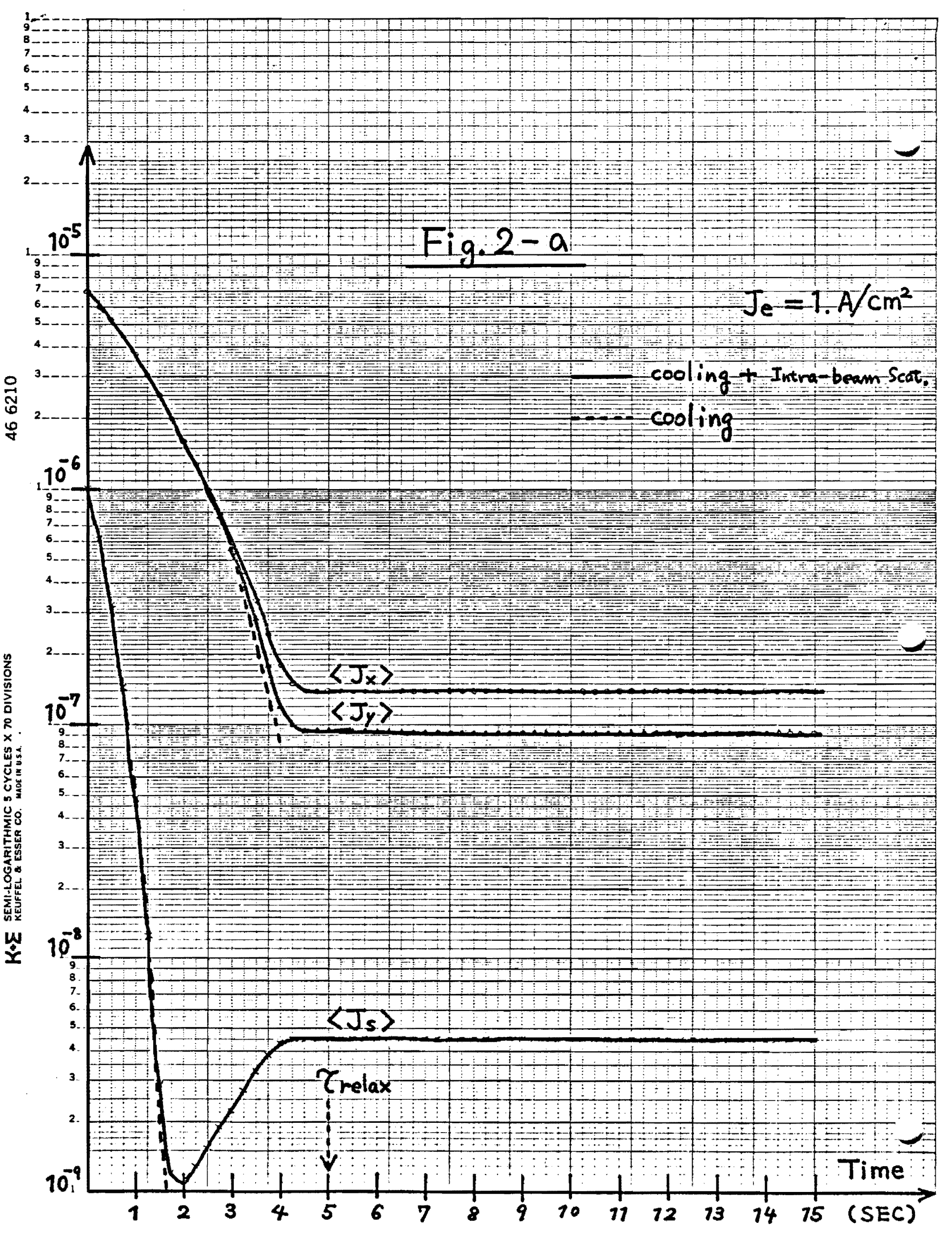




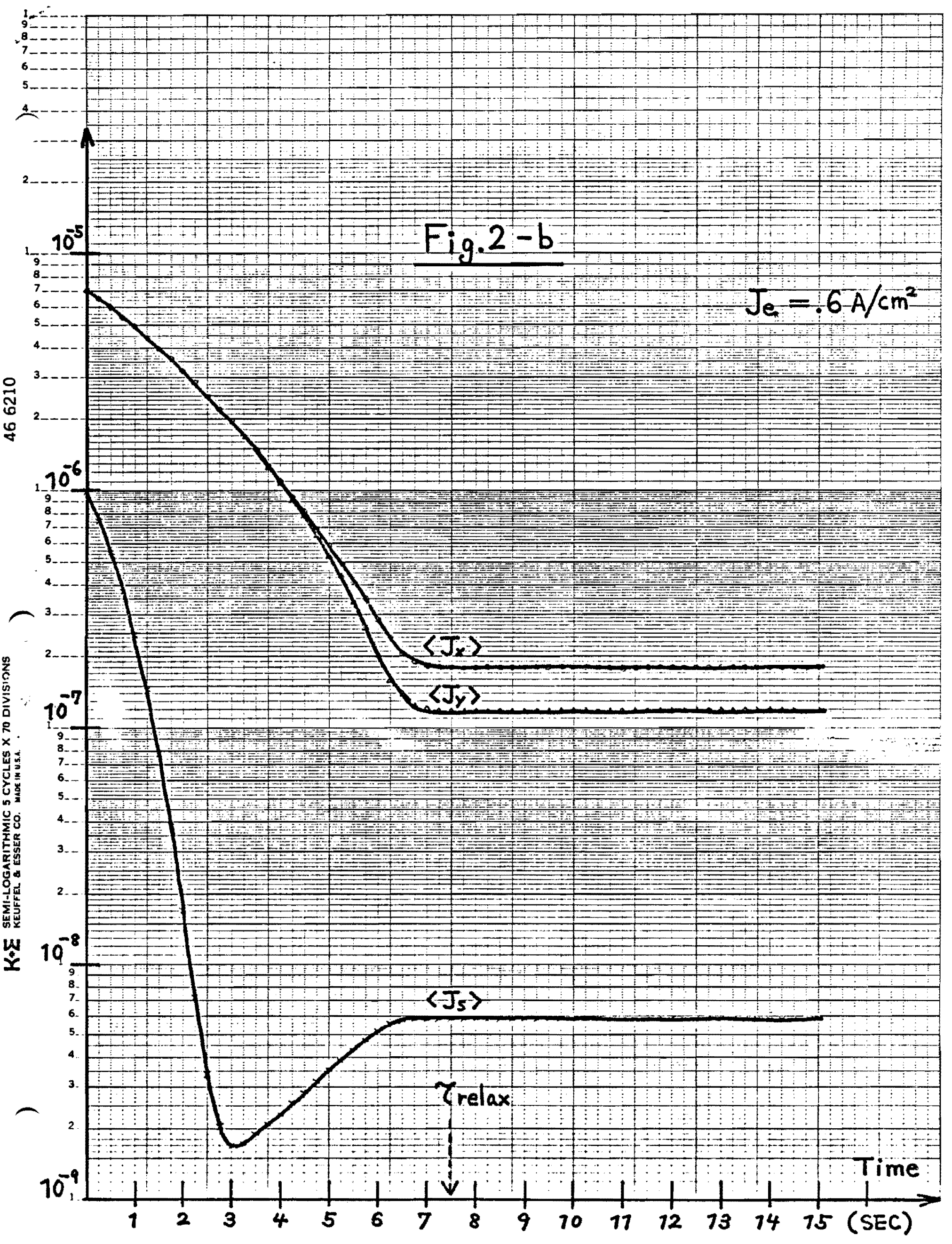




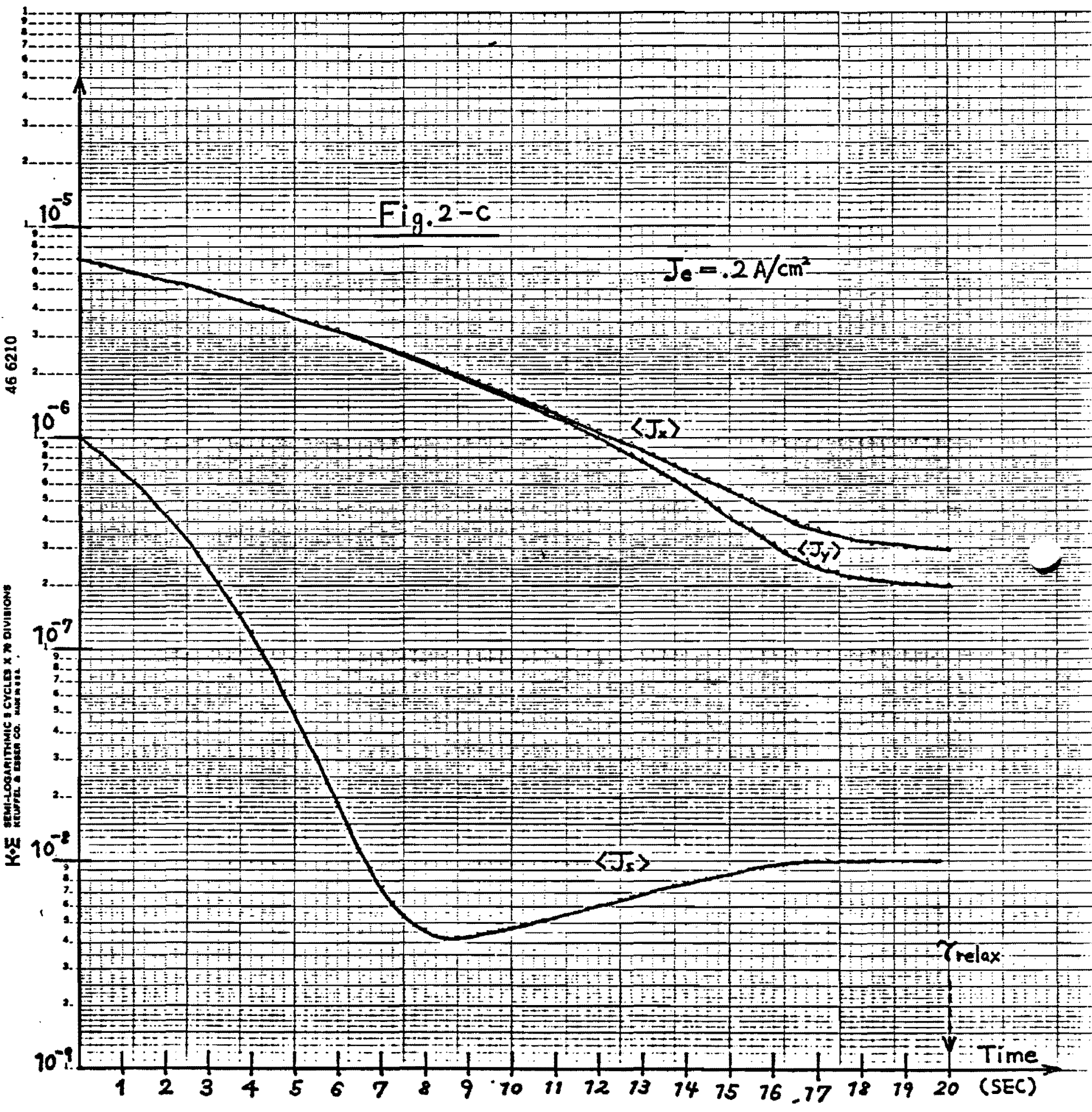




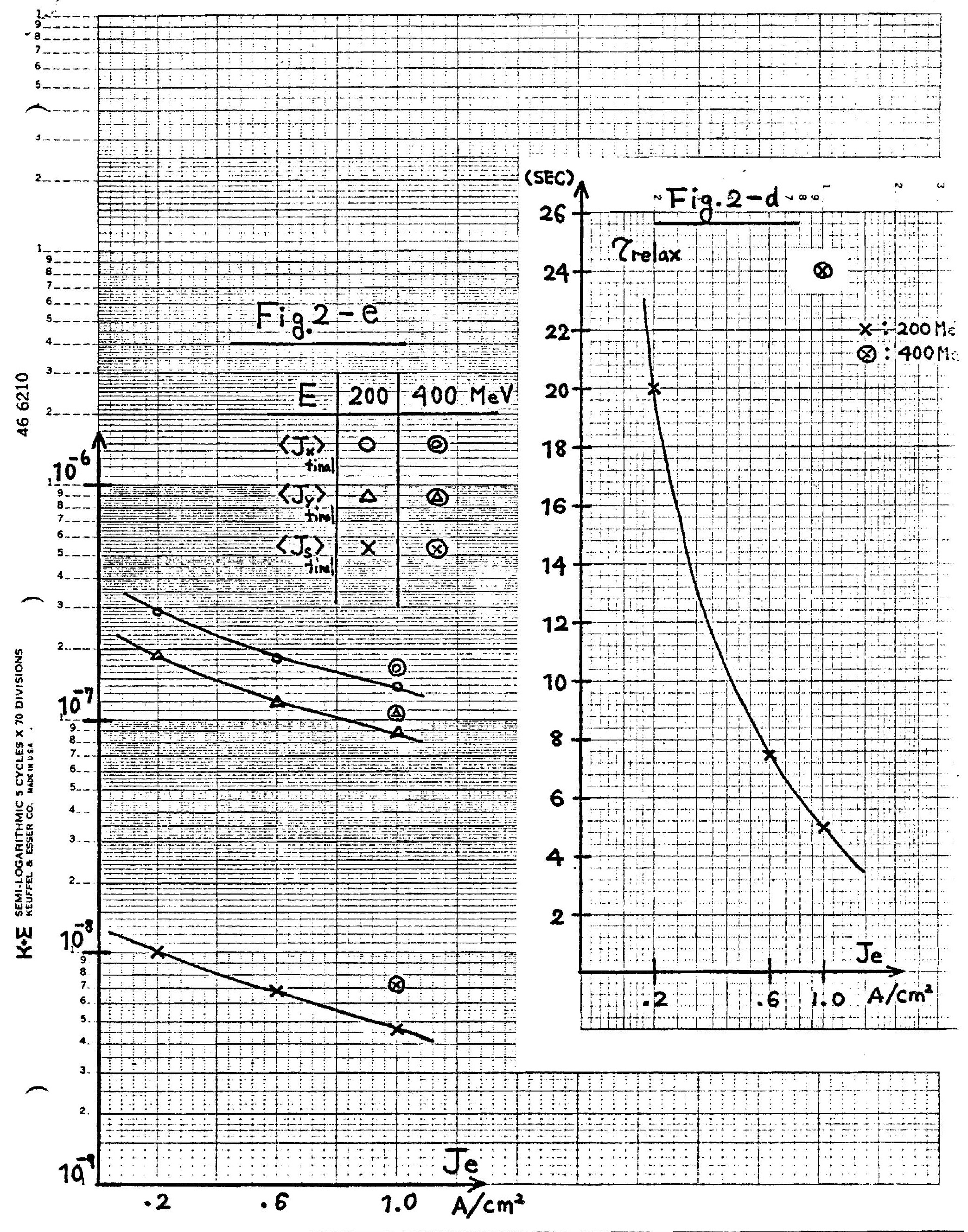

